\title{
Dopant-Free Hole Transport Materials with a Long Alkyl Chain for Stable Perovskite Solar Cells
}

\author{
Kai Wang ${ }^{1,+}$, Haoran Chen ${ }^{1,+}$, Tingting Niu ${ }^{1}$, Shan Wang ${ }^{1}$, Xiao Guo ${ }^{1}$ and Hong Wang ${ }^{2, *}$ (D) \\ 1 Key Laboratory of Flexible Electronics (KLOFE) \& Institute of Advanced Materials (IAM), Jiangsu National \\ Synergetic Innovation Center for Advanced Materials (SICAM), Nanjing Tech University (Nanjing Tech), \\ Nanjing 211816, China \\ 2 Zhongshan Institute of Modern industrial Technology, South China University of Technology, Zhongshan \\ 528437, China \\ * Correspondence: phhwang@scut.edu.cn; Tel.: +86-136-0006-6193 \\ + These authors contributed equally to this work.
}

Received: 22 May 2019; Accepted: 24 June 2019; Published: 28 June 2019

check for updates

\begin{abstract}
Hole transport materials are indispensable to high efficiency perovskite solar cells. Two new hole transporting materials (HTMs), named 4,4'-(9-nonyl-9H-carbazole-3,6-diyl)bis (N,N-bis(4-methoxyphenyl)aniline) (CZTPA-1) and 4,4'-(9-methyl-9H-carbazole-3,6-diyl)bis (N,N-bis(4-methoxyphenyl)aniline)(CZTPA-2), were developed by different alkyl substitution methods. The two compounds, containing a carbazole core and triphenylamine (TPA) groups with different lengths of the alkyl chain, were designed and synthesized through a two-step synthesis approach. The power conversion efficiency (PCE) was found to be affected by the length of the alkyl chain, reaching 7\% for CZTPA-1 and 11\% for CZTPA-2. Furthermore, the CZTPA-2 still maintained $89.7 \%$ of its original performance after $400 \mathrm{~h}$. The proposed results demonstrate the effect of carbon chain substituents on the efficiency of perovskite solar cells (PSCs).
\end{abstract}

Keywords: perovskite solar cell; alkyl chain; hole transporting materials; stable

\section{Introduction}

Perovskite solar cells (PSCs) have attracted much attention in recent years owing to their excellent optoelectronic properties, high absorption coefficients, easy solution processability, high carrier mobility, and so on [1-5]. Many efforts have been made to increase the efficiency of PSCs. Surprisingly, it has increased dramatically from $3.8 \%$ [1] to $24.2 \%$ [6] in a short period of time.

As is well-known, 2,2',7,7'-Tetrakis[N,N-di(4-methoxyphenyl)amino]-9,9'-spirobifluorene (Spiro-OMeTAD) [7,8] is a common hole transporting material (HTM) with good performance. However, it is still a formidable challenge to develop this material due to various drawbacks, such as a complicated synthesis process and high cost. Therefore, new HTMs with a simple synthesis process and low cost need urgently to be developed.

HTMs construct the hole transport layer (HTL) that is needed to block electron transport, enhance hole transport, and prevent direct contact between the perovskite layer and the electrode, which causes annihilation. An ideal HTM should have such characteristics as good hole mobility, good hydrophobicity, suitable energy levels, and the ability to be prepared in solution [9-11]. Currently, common HTMs can be classified as inorganic substances, organic polymers, or organic small molecules depending on the type of material. Low cost, high stability, and hole mobility are among the many advantages that HTMs based on inorganic materials have. Kamat and co-workers first introduced copper iodide as an HTM and achieved an average PCE of 6\% [12]. Then, inorganic semiconductors (CuSCN, NiO, CuI) as HTMs were developed [13,14]. In addition to inorganic HTMs, polymer-based 
HTMs have been explored in PSCs. Poly-[bis(4-phenyl)(2,4,6-trimethylphenyl)amine] (PTAA) was the first conjugated polymer to be used as an HTM in PSCs [15] and maintained the highest PCE of any reported polymeric HTM [16]. Accompanied by the development of conjugated polymer HTMs, Poly(3-hexylthiophene-2,5-diyl (P3HT) [17] was originally used in organic solar cells (OSCs) as the active layer was introduced into the hole transport layer. Compared to polymeric HTMs, small molecule HTMs have the advantages of a determined molecular weight and simple purification for PSCs. As a group with good stability and solubility, triphenylamine (TPA) is widely used in organic small molecule HTMs [18-20]. The TPA group in particular can influence the optoelectrical properties of HTMs due to its non-planar geometry. Furthermore, the carbazole group has a high carrier mobility in OSCs and as good a performance as HTMs; thus, it is currently regarded as a core in PSCs [21-27].

In 2014, Sun and co-workers [27] designed a series of HTMs based on carbazole, named X19 and X51. The X51 realized a PCE of $9.8 \%$ due to a higher charge-carrier mobility and conductivity than X19. Subsequently, Nazeeruddin and co-workers [28] studied bridged carbazole with biphenyl, by using silolothiophene as the bridge, which obtained a PCE of $13.1 \%$. They found that compared to the spirofluorene-linked triphenylamine HTMs, novel silolothiophene-linked methoxy triphenylamines (Si-OMeTPAs) enable more stable PSCs. In the same year, Tang and co-workers [29] used carbazole as a core with four TPAs as the side groups to achieve a PCE of up to $18.32 \%$.

Recently, Khaja Nazeeruddin and co-workers [30] used anthra[1,2-b:4,3-b':5,6-b':8,7$\mathrm{b}^{\prime \prime \prime}$ ]tetrathiophene as the core, by changing the alkyl chain length of the methoxy groups on the triarylamine sites, to develop a series of materials. The device based on a methyl substitute named ATT-OMe was found to have the best PCE of $18.13 \%$, which was better than that of the device based on other longer alkyl chain HTMs. They believed that the presence of alkyl chains decreased the hole-transport properties. However, the performance of PSCs based on carbazole is far from that of the classical Spiro-OMeTAD. Therefore, more efforts are needed to develop new small molecule HTMs that match with perovskite instead of the Spiro-OMeTAD.

In this work, we developed two HTMs-4,4'-(9-nonyl-9H-carbazole-3,6-diyl)bis (N,N-bis(4-methoxyphenyl)aniline) (CZTPA-1) and 4,4'-(9-methyl-9H-carbazole-3,6-diyl)bis (N,N-bis(4-methoxyphenyl)aniline) (CZTPA-2) - based on TPA as the end group. They both have the advantage of simple synthesis steps and low cost. Both HTMs are synthesized by one-step Suzuki coupling. The cost of the raw material 3,6-Dibromocarbazole $(\$ 0.3 / \mathrm{g})$ plus 4-methoxy- $N$-(4-methoxyphenyl)- $N$-(4-(4,4,5,5-tetramethyl-1,3,2-dioxaborolan-2-yl)phenyl)aniline $(\$ 15 / \mathrm{g})$ is clearly lower than that of Spiro-OMeTAD (\$220/g). Notably, the CZTPA-2 with the longer alkyl chain achieved a better PCE of $11.79 \%$ with a short current density $\left(J_{\mathrm{sc}}\right)$ of $21.80 \mathrm{~mA} / \mathrm{cm}^{2}$, an open circuit voltage $\left(V_{\mathrm{oc}}\right)$ of $0.99 \mathrm{~V}$, and a fill factor $(\mathrm{FF})$ of $54.59 \%$. This is attributed to the significantly improved hole mobility of CZTPA-2, resulting in a significant increase in device efficiency.

\section{Materials and Methods}

\subsection{Materials}

Unless otherwise noted, all reagents used in the experiments were purchased from commercial sources and used without further purification. 3,6-Dibromo-9H-carbazole, $\mathrm{N}, \mathrm{N}$-bis(4-Methoxyphenyl)-4-(4,4,5,5-tetraMethyl-1,3,2-dioxaborolan-2-yl)-BenzenaMine, lead iodide $\left(\mathrm{PbI}_{2}\right)$, methylammonium iodide (MAI), acetonitrile $(99.8 \%)$, chlorobenzene $(99.9 \%)$, and dimethylformamide (DMF) (99\%) were purchased from Sigma-Aldrich. 4-tert-butylpyridine (TBP) and Li-bis-(trifluoromethanesulfonyl) imide (Li-TFSI) were purchased from TCI. 2,2',7,7'-tetrakis-( $N, N$-di-p-methoxyphenylamine)-9,9'-spirobifluorene (Spiro-OMeTAD) (99.0\%) was purchased from Xi'an Polymer Light Technology Co., Ltd.

Perovskite precursor: The perovskite precursor was obtained by mixing $\mathrm{PbI}_{2}$ and $\mathrm{MACl}$ (in a molar ratio of 1:1) in DMF with a concentration of $350 \mathrm{mg} / \mathrm{mL}$, and was then stirred at $60{ }^{\circ} \mathrm{C}$ overnight in a glovebox. 
Spiro-OMeTAD: The 2,2',7,7'-Tetrakis( $N, N^{\prime}$-di-p-methoxyphenylamine)-9, $9^{\prime}$-spirobifluorene (Spiro-OMeTAD) was doped with TBP and Li-TFSI. A total of $73.2 \mathrm{mg}$ of Spiro-OMeTAD (Xi'an Polymer Light Technology Co., Ltd., Xi'an, China) was dissolved in $1 \mathrm{~mL}$ of chlorobenzene (CB) with $28.8 \mu \mathrm{L}$ of 4-tert-butylpyridine (TBP) and $17.6 \mu \mathrm{L}$ of Li-bis-(trifluoromethanesulfonyl) imide (Li-TFSI).

\subsection{Device Fabrication}

The $\mathrm{SnO}_{2}$ layer was spin-coated on an ITO substrate at $3000 \mathrm{rpm}$ for $30 \mathrm{~s}$, which was cleaned in $\mathrm{UV-ozone}$ and then annealed at $150^{\circ} \mathrm{C}$ for $30 \mathrm{~min}$. The perovskite layer was spin-coated at $4000 \mathrm{rpm}$ for $30 \mathrm{~s}$ by an anti-solvent method. The details of the operation are as follows: $100 \mu \mathrm{L} \mathrm{CB}$ was rapidly added after $5 \mathrm{~s}$ of spin-coating with perovskite solution; and the perovskite films were annealed at $100{ }^{\circ} \mathrm{C}$ for $5 \mathrm{~min}$. All of the above processes were performed in the nitrogen glovebox. Then, Spiro-OMeTAD and two HTMs were dissolved in the CB $(10 \mathrm{mg} / \mathrm{mL})$, and then spin-coated upon the perovskite layer at $3000 \mathrm{rpm}$ for $30 \mathrm{~s}$. Then, molybdenum trioxide $\left(\mathrm{MoO}_{3}\right)$ and gold $(\mathrm{Au})$ were thermally evaporated on the hole transporting layer. The effective area of the cell is $0.05 \mathrm{~cm}^{2}$.

\subsection{Device Characterization}

The cross-sectional images of PSC were taken by scanning electron microscopy (SEM) (ZEISS Merlin, Carl Zeiss Microscopy, Jena, Germany). The UV-visible absorption spectra were measured using a UV Spectrophotometer (SHIMADZU UV-1750, East Test Technology Co., Ltd, Shenzhen, China). Photoluminescence (PL) spectra were obtained using a spectrofluorometer (HitachiF-7000, Hitachi High-Technologies Corporation, Shenzhen, China). Thermogravimetric (TGA) analysis was performed on a Mettler Toledo TGA2.

The device was measured under AM $1.5 \mathrm{G}$ solar irradiation with an intensity of $100 \mathrm{~mW} / \mathrm{cm}^{2}$ through an Enlitech SS-F5-3A solar simulator. The instrument was calibrated on standard solar cells. $J-V$ properties were measured by the method of Enlitech Ltd. (Kaohsiung, Taiwan) and a Keithley (Cleveland, OH, USA) 2400 source meter under dark conditions. The external quantum efficiency (EQE) spectra were measured using a solar cell IPCE test system (CROWNTECH Inc., model QTEST HIFINITY (Macungie, PA, USA)).

The synthesis method and details of the experimental procedure are shown in the supporting information (SI).

\section{Results}

The details of the experimental procedure are shown in the Supplementary Information (SI). The design principle was to improve planarity as well as increase solubility. The carbazole group with simple structures has good hole transporting ability. By inserting $\mathrm{N}$ atoms with different alkyl chain lengths, the optoelectronic properties and solubility can be adjusted. Herein, we chose 4-methoxy- $N$-(4-methoxyphenyl)- $N$-(4-(4,4,5,5-tetramethyl-1,3,2-dioxaborolan-2-yl)phenyl)aniline units as the raw material, used Suzuki coupling, and substituted the carbazole core with different lengths of alkyl chains. The aimed-for materials were obtained by simple column chromatography separation and recrystallization.

Figure 1a shows the absorption spectra of CZTPA- 1 and CZTPA-2 in chloroform and as a coating on quartz substrates. Absorption peaks at $335 \mathrm{~nm}$ for CZTPA- 1 and $327 \mathrm{~nm}$ for CZTPA-2 in the solution were observed. Relative to the solution, the CZTPA- 1 film exhibits a redshift of $2 \mathrm{~nm}$ with an onset of $409 \mathrm{~nm}$, corresponding to an optical bandgap of $3.03 \mathrm{eV}$, whereas CZTPA-2 aligns to a narrower bandgap of $2.98 \mathrm{eV}$ (onset of $416 \mathrm{~nm}$ ). Both HTMs have a slight redshift in the film compared with the solution, suggesting that aggregation exists in the films. 
a

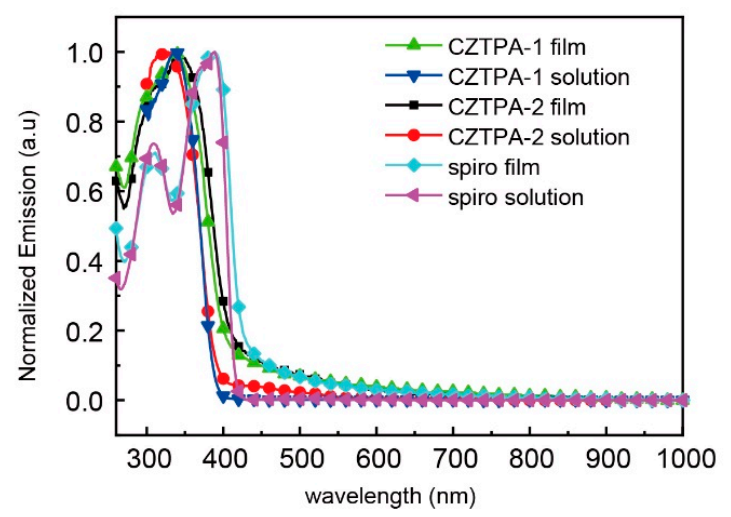

b

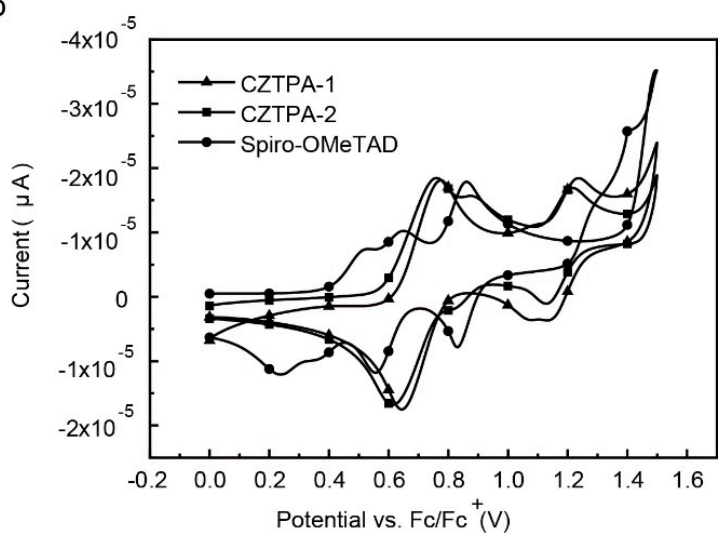

Figure 1. (a) Optical absorption spectra of 4,4'-(9-nonyl-9H-carbazole-3,6-diyl)bis (N,N-bis(4-methoxyphenyl)aniline) (CZTPA-1) and 4,4'-(9-methyl-9H-carbazole-3,6-diyl)bis ( $N, N$-bis(4-methoxyphenyl)aniline) (CZTPA-2) in chloroform and thin films spin-coated from chloroform. (b) Cyclic voltammetry of CZTPA-1 and CZTPA-2.

From Figure 1b, the highest occupied molecular orbital (HOMO) level of CZTPA-1 is $-4.89 \mathrm{eV}$, while in the CZTPA-2 there is a higher HOMO of $-4.83 \mathrm{eV}$ (the energy level of Ferroceneis is $-0.54 \mathrm{eV}$ by the cyclic voltammetry method). Compared to the two HTMs, the Spiro-OMeTAD has the higher energy level as the $\mathrm{HOMO}$ is $-4.64 \mathrm{eV}$. Based on the relationship of $E_{\mathrm{LUMO}}=E_{\mathrm{HOMO}}+E_{\mathrm{g}}$, the lowest unoccupied molecular orbital (LUMO) level value was calculated to be $-1.86 \mathrm{eV}$ for CZTPA-1, -1.85 eV for CZTPA-2, and -1.74 eV for Spiro-OMeTAD (Table 1). CZTPA-2 has similar electrochemical properties compared with CZTPA-1, which indicates that the electrochemical properties of the molecule remain stable with a change in the alkyl chain length.

Table 1. Optical and electrochemical properties of CZTPA-1 and CZTPA-2.

\begin{tabular}{ccccccc}
\hline & $\begin{array}{c}\lambda_{\max } \text { Sol (a) } \\
(\mathbf{n m})\end{array}$ & $\begin{array}{c}\lambda_{\max } \text { Film (b) } \\
(\mathbf{n m})\end{array}$ & $\begin{array}{c}\lambda_{\text {onset }} \\
(\mathbf{n m})\end{array}$ & $\begin{array}{c}E_{\mathbf{g}} \mathbf{\text { opt }}(\mathbf{c}) \\
(\mathbf{e V})\end{array}$ & $\begin{array}{c}E_{\text {HOMO }} \\
(\mathbf{e V})\end{array}$ & $\begin{array}{c}E_{\text {LUMO }}(\mathbf{d}) \\
(\mathbf{e V})\end{array}$ \\
\hline CZTPA-1 & 335 & 337 & 409 & 3.03 & -4.89 & -1.86 \\
CZTPA-2 & 327 & 339 & 416 & 2.98 & -4.83 & -1.85 \\
Spiro-OMeTAD & 386 & 390 & 428 & 2.90 & -4.64 & -1.74 \\
\hline
\end{tabular}

(a) Maximum absorption peak in $\mathrm{CH}_{2} \mathrm{Cl}_{3}$ solution; (b) Maximum absorption peak of films on quartz glass; (c) Optical bandgap calculated from the absorption onset of films: $E_{\mathrm{g}}{ }^{\mathrm{opt}}=1240 / \lambda_{\text {onset }} \mathrm{eV} ;(\mathrm{d}) E_{\mathrm{LUMO}}=E_{\mathrm{HOMO}}+E_{\mathrm{g}}{ }^{\text {opt }}$.

We then found that CZTPA-1 and CZTPA-2 match well with the energy level of the perovskite from Figure 2a. Furthermore, the thermal stability of the two HTMs was measured by thermogravimetric analysis (TGA) (Figure 2b). Both CZTPA-1 and CZTPA-2 exhibit good thermal stability with decomposition temperatures $\left(\mathrm{T}_{\mathrm{d}}, 5 \%\right.$ weight loss) at $391.8{ }^{\circ} \mathrm{C}$ and $384.6{ }^{\circ} \mathrm{C}$, respectively. As we expected, increasing the length of the alkyl chains reduces the thermal stability.

The photoluminescence (PL) spectra in Figure 2c show the maximum emission peak at $431 \mathrm{~nm}$ for CZTPA-1 and at $425 \mathrm{~nm}$ for CZTPA-2 in film. CZTPA-2 was slightly more blue-shifted than CZTPA-1, caused by the increase in the length of the alkyl chains.

Figure $2 \mathrm{~d}$ shows the PL spectra of the perovskite, perovskite with Spiro-OMeTAD, perovskite with CZTPA-1, and perovskite with CZTPA-2. Strong PL quenching was observed after the HTMs were coated on perovskite films. Respectively, compared with the original perovskite film, the PL intensity was reduced to $7 \%, 22 \%$, and $18 \%$ after coating with Spiro-OMeTAD, CZTPA-1, and CZTPA-2. Thus, we think that CZTPA-2 has better charge separation than CZTPA-1 and a smaller $J_{S C}$ and FF in the PSCs compared to Spiro-OMeTAD devices. In short, this means that the hole transfer capabilities of CZTPA-2 are superior because of their better charge transfer capability. 
a
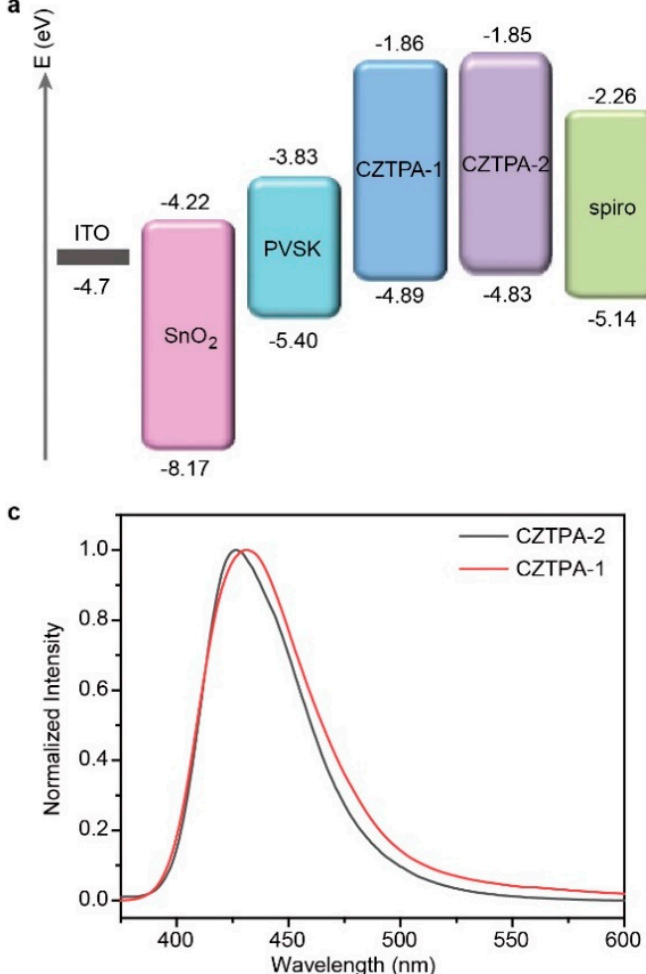
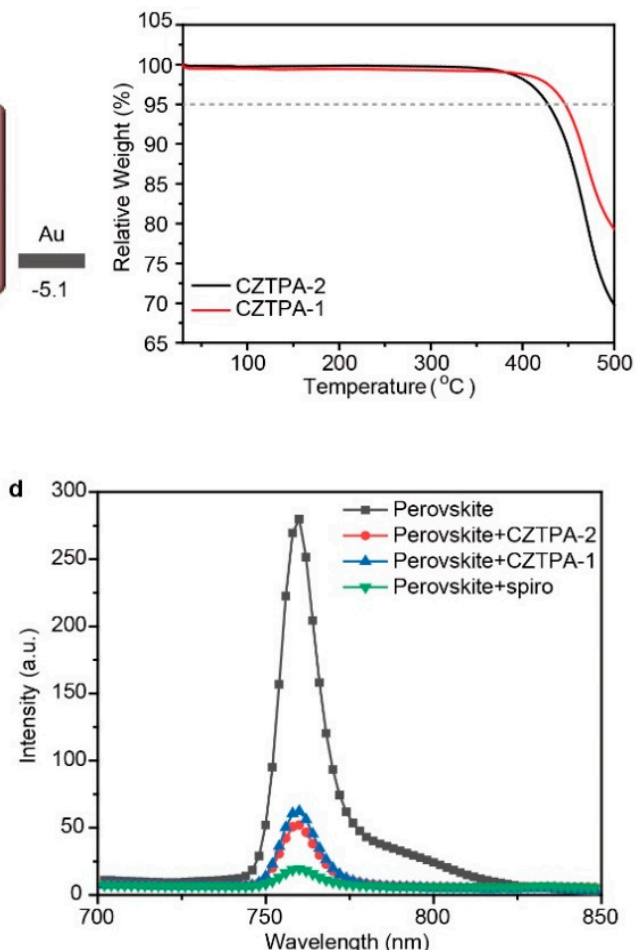

Figure 2. (a) Energy level diagram of a perovskite solar cell (PSC) with CZTPA-1, CZTPA-2, and $2,2^{\prime}, 7,7^{\prime}$-Tetrakis[N,N-di(4-methoxyphenyl)amino]-9,9'-spirobifluorene (Spiro-OMeTAD). (b) Thermogravimetric analysis (TGA) diagrams of CZTPA-1 and CZTPA-2. (c) Photoluminescence (PL) spectra of CZTPA-1 and CZTPA-2 thin film, excitation at $350 \mathrm{~nm}$. (d) Photoluminescence spectra of perovskite, perovskite with Spiro-OMeTAD, perovskite with CZTPA-1, and perovskite with CZTPA-2, excitation at $500 \mathrm{~nm}$.

Figure 3 shows two cross-sectional scanning electron microscopy (SEM) images of the PSC with the structure of ITO/SnO $/$ perovskite/HTMs/Au. The PSC includes an $\approx 460 \mathrm{~nm}$ perovskite capping layer and an $\approx 35 \mathrm{~nm}$ HTM layer (CZTPA-1 or CZTPA-2). From the SEM image, we found that the HTM layer deposited on the perovskite layer and the boundaries of each layer are clear. Atomic force microscopy (AFM) images of the two materials spin-coated onto perovskite films are shown in Figure S7, which represent the perovskite/CZTPA-2 (a) and the perovskite/CZTPA-1 (b) films, respectively. The roughness of both samples is slightly high, which may be due to the lower thickness of the HTMs. After a comparison, it was found that the roughness of the film in which the HTM is CZTPA-2 (RMS = $20.968 \mathrm{~nm}$ ) is significantly lower than that of CZTPA-1 (RMS = $28.662 \mathrm{~nm})$; this is attributed to the solubility of CZTPA-2 being higher such that it could better cover the film and further improve the carrier transport of the device.
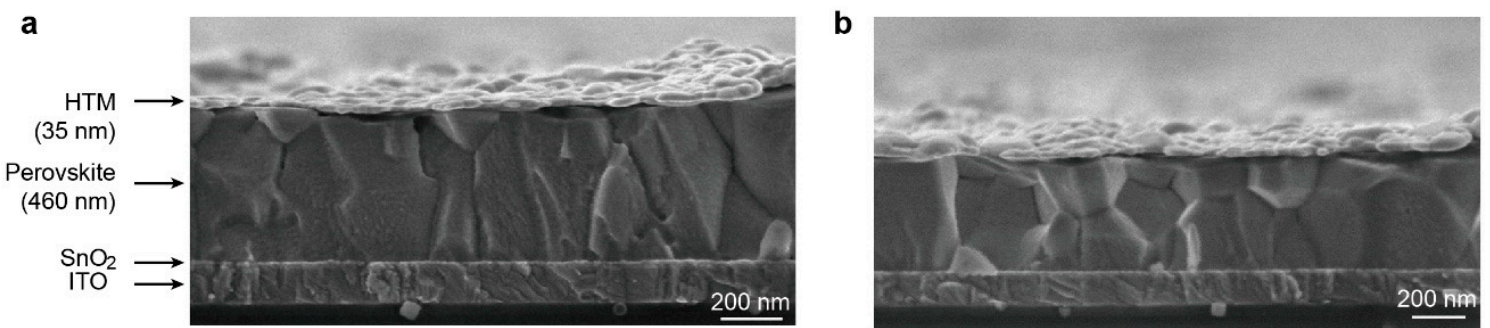

Figure 3. (a) A cross-sectional SEM image of the CZTPA-1 PSC. The scale bar is $200 \mathrm{~nm}$. (b) A cross-sectional SEM image of the CZTPA-2 PSC. The scale bar is $200 \mathrm{~nm}$. 
In order to compare the properties of the two HTMs, we used them as the hole transport layer of perovskite solar cells to compare device performance. Between them, the effective area of the cells is $0.05 \mathrm{~cm}^{2}$, and the scanning rate is $0.02 \mathrm{~V} / \mathrm{s}$. As shown in Figure 4a,b, the PSCs with CZTPA-2 achieve a PCE of $11.79 \%$ with an open-circuit voltage $\left(V_{\mathrm{oc}}\right)$ of $0.99 \mathrm{~V}$, a short-circuit current density $\left(J_{\mathrm{sc}}\right)$ of $21.8 \mathrm{~mA} \mathrm{~cm}^{-2}$, and a fill factor (FF) of $54.59 \%$, while the PSCs with CZTPA-1 achieve a lower PCE of $6.05 \%$ under the condition of no doping. In contrast, the best cell is based on Spiro-OMeTAD, as the HTM achieves the PCE of $16.77 \%$. We also compared the Spiro-OMeTAD PSC without any doping, which achieved a PCE of $11.74 \%$. The photovoltaic performance of the PSCs based on the two HTMs (dopant-free) and the Spiro-OMeTAD were investigated under AM 1.5 illumination $\left(100 \mathrm{~mW} \mathrm{~cm}^{-2}\right)$. The champion device performance plots of CZTPA-1 and CZTPA-2 are shown in Table 2. The device fabricated with CZTPA-1 as the HTM yielded a promising PCE of $6.05 \%$ with a $J_{\mathrm{sc}}$ of $20.58 \mathrm{~mA} \mathrm{~cm}^{-2}$, a $V_{\text {oc }}$ of $0.77 \mathrm{~V}$, and an FF of 38.01\%. We calculated the average PCE of the CZTPA-2 devices to be $10.15 \% \pm 0.90$. The CZTPA-1 devices own an average PCE of $5.27 \% \pm 0.57$. The average PCE of the Spiro-OMeTAD (dopant-free) is $10.02 \% \pm 0.98$, and the corresponding PCE of Spiro-OMeTAD is $15.65 \% \pm 0.71$. All data are based on the values obtained from 20 devices. From these, we can speculate that CZTPA-2 exhibits better performance compared with CZTPA-1. The FF and $V_{\text {oc }}$ of CZTPA-1 are obviously lower than those of CZTPA-2 in the PSC devices. Furthermore, CZTPA-2 obtains a slightly higher PCE compared with the dopant-free Spiro-OMeTAD, which is attributed to the higher $J_{\mathrm{sc}}$.

a
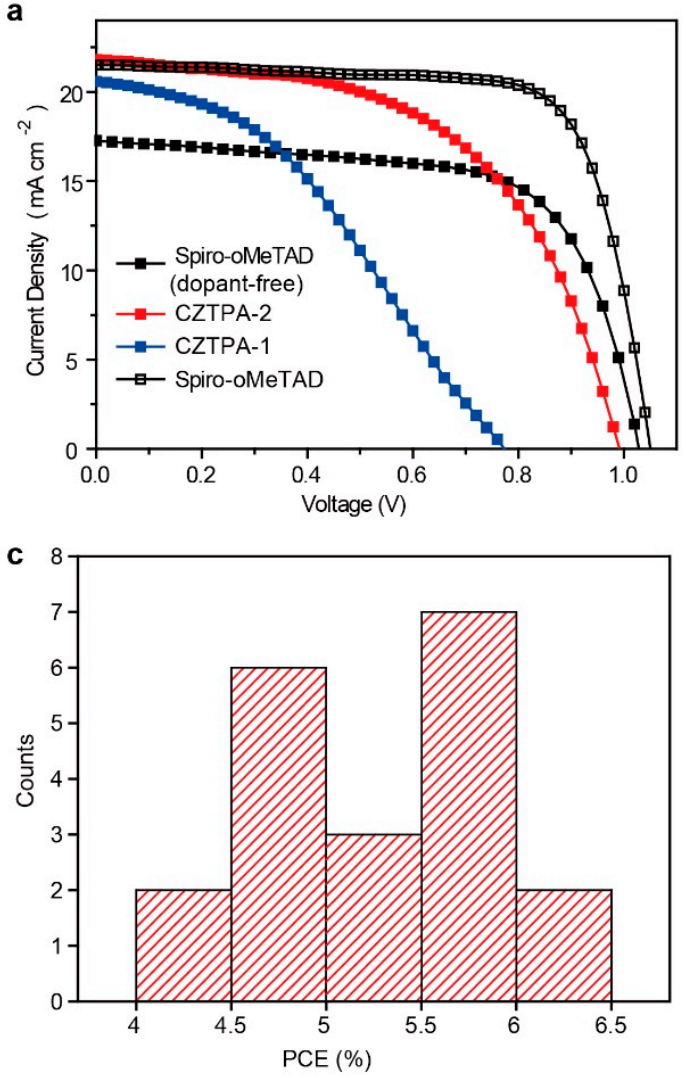

b

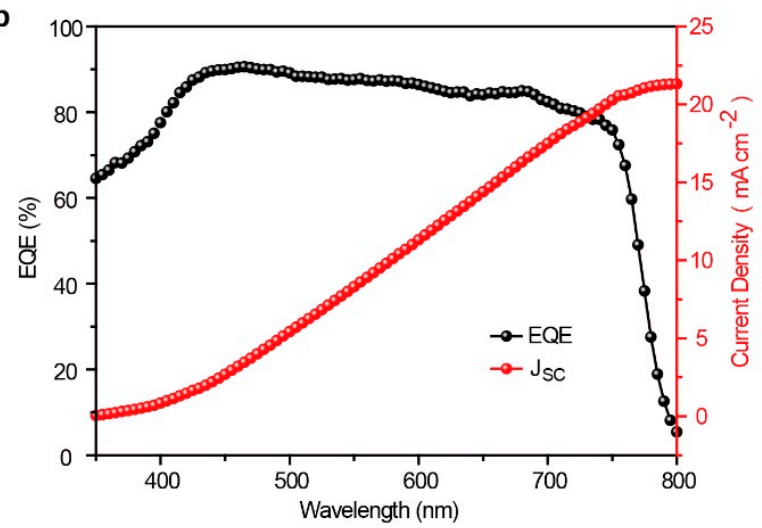

d

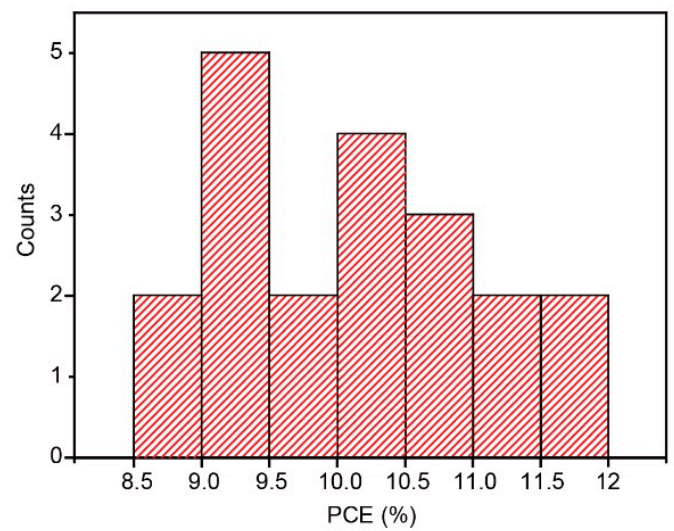

Figure 4. (a) $J-V$ characteristics of PSCs based on the two hole transport materials (HTMs), the Spiro-OMeTAD, and the Spiro-OMeTAD (dopant-free). (b) External quantum efficiency (EQE) and $J_{\text {sc }}$ spectra of PSCs with CZTPA-2. (c) Histograms of PCEs measured in 20 cells of CZTPA-1. (d) Histograms of PCEs measured in 20 cells of CZTPA-2. 
Table 2. Photovoltaic data of PSCs based on the two HTMs, the Spiro-OMeTAD, and the Spiro-OMeTAD (dopant-free).

\begin{tabular}{cccccc}
\hline & $V_{\text {OC }}(\mathbf{V})$ & $J_{\text {SC }}\left(\mathbf{m A} \cdot \mathbf{c m}^{-\mathbf{2}}\right)$ & FF (\%) & PCE (\%) & PCE Ave (\%) \\
\hline Spiro-OMeTAD (dopant-free) & 1.02 & 17.26 & 66.14 & 11.74 & $10.02 \% \pm 0.98$ \\
CZTPA-2 & 0.99 & 21.80 & 54.59 & 11.79 & $10.15 \% \pm 0.90$ \\
CZTPA-1 & 0.77 & 20.58 & 38.01 & 6.05 & $5.27 \% \pm 0.57$ \\
Spiro-OMeTAD & 1.05 & 21.51 & 74.24 & 16.77 & $15.65 \% \pm 0.71$ \\
\hline
\end{tabular}

When preparing the solution, we found the CZTPA- 1 dissolution rate to be slower than the CZTPA-2 dissolution rate. What is more, the solubility of CZTPA-2 was found to be better than that of CZTPA-1 with an increasing alkyl chain length and CZTPA-2 deposits in perovskite with better crystallization. These results also demonstrate that CZTPA-2 achieves better performance.

To test the reproducibility of the devices, we fabricated 20 devices in several different batches. The devices are shown in Figure 4c,d. As shown in the PCE histogram of the corresponding device data, the average PCE of CZTPA- 2 and CZTPA- 1 is $10.15 \%$ and $5.27 \%$, respectively.

Moreover, the EQE spectrum of PSCs with CZTPA-2 is also shown in Figure $4 \mathrm{~b}$. The integral of the current densities calculated from the EQE spectra is $21.32 \mathrm{~mA} \mathrm{~cm}{ }^{-2}$ for CZTPA-2 according predominantly to the experimental data.

To calculate the hole mobility of the two HTMs, we constructed a device with a configuration of ITO/PEDOT:PSS/HTM/Au using the space-charge-limited current (SCLC) method, and the J- $V$ characteristics of this device were studied in the dark. Hole mobility is calculated by the Mott-Gurney equation of $J=9 \varepsilon_{\mathrm{r}} \varepsilon_{0} \mu \mathrm{Va}^{2} / 8 \mathrm{~L}^{3}$, so we change the form of the formula to obtain $\mu=8 \mathrm{~d}^{3} / 9 \varepsilon_{\mathrm{r}} \varepsilon_{0}\left(J^{1 / 2} / \mathrm{Va}\right)^{2}$, where $\varepsilon_{\mathrm{r}}$ is the relative dielectric constant of the transport medium $\left(\varepsilon_{\mathrm{r}}=3\right.$ for organic materials), $\varepsilon_{0}$ is the permittivity of free space $\left(8.85 \times 10^{-12} \mathrm{C} \mathrm{V}^{-1} \mathrm{~m}^{-1}\right)$, $J$ is the dark current density $\left(\mathrm{mA} \mathrm{cm}^{-2}\right)$, and $\mathrm{d}$ is the thickness of the active layer [31]. $\mathrm{d}$ is $48 \mathrm{~nm}$ for CZTPA-1 and $56 \mathrm{~nm}$ for CZTPA-2. Figure 5 shows that the hole mobility of Spiro-OMeTAD (doped) is $1.01 \times 10^{-3} \mathrm{~cm}^{2} \mathrm{~V}^{-1} \mathrm{~s}^{-1}$. The hole mobility of CZTPA-1 is $4.68 \times 10^{-5} \mathrm{~cm}^{2} \mathrm{~V}^{-1} \mathrm{~s}^{-1}$, and CZTPA-2 has the higher hole mobility of $8.06 \times 10^{-5} \mathrm{~cm}^{2}$ $\mathrm{V}^{-1} \mathrm{~s}^{-1}$. However, they are all lower than the hole mobility of Spiro-OMeTAD (doped). Compared to CZTPA-1, CZTPA-2 has a higher hole mobility, which leads to good hole transport and enhances the charge transport in a planar PSC. CZTPA-2's higher hole mobility can be attributed to its high hole transport capability. These results suggest that both a fast charge transfer and high hole transport capability contribute to a high PCE.
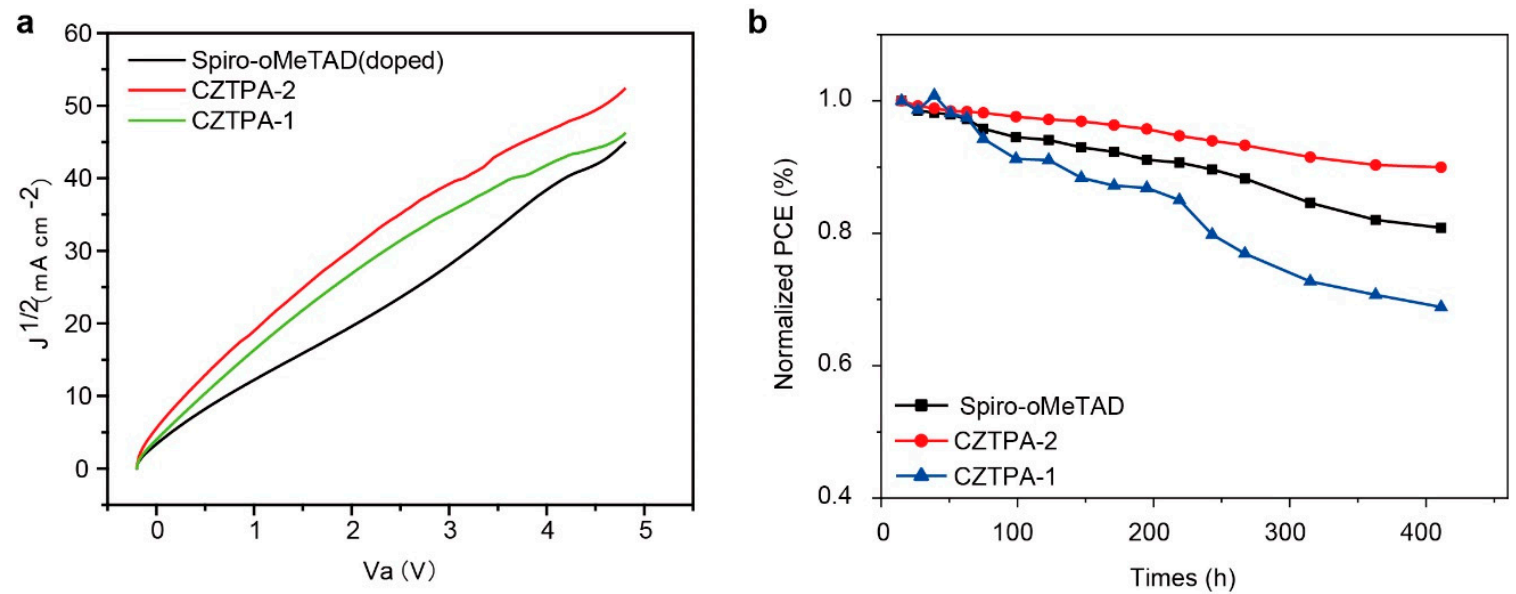

Figure 5. (a) Space-charge-limited current (SCLC) $J^{1 / 2}-V$ characteristics of CZTPA-1-, CZTPA-2-, and Spiro-OMeTAD-based hole-only devices measured in the dark. (b) Stability of CZTPA-1, CZTPA-2, and Spiro-oMeTAD (dopant-free) without encapsulation. 
We tested the stability of the three HTM devices without encapsulation by storing them in the dark under air conditions for at least $400 \mathrm{~h}$. The PCE over time curve was plotted and is shown in Figure 5b. The PCE of the CZTPA-2 device was still over 10\%. In comparison, the PCE of Spiro-OMeTAD (dopant-free) device dropped below 10\%. CZTPA-1 exhibited low performance, with $68.2 \%$ of the original PCE. CZTPA-2 and Spiro-OMeTAD (dopant-free) maintained $89.7 \%$ and $81.6 \%$ of their initial PCE, respectively. This test verified that the device based on CZTPA-2 has the best stability of the three HTMs. The long alkyl chain, which has good morphology, may influence the stability of a PSC device.

Finally, we also tested the capacitance of the three HTMs. The capacitance versus frequency was plotted and is shown in Figure 6. The capacitance is mainly caused by charge or ion accumulation at the perovskite interface, which leads to interfacial recombination. We can see the capacitance of the device based on CZTPA-2 is obviously smaller than that of CZTPA-1 and Spiro-oMeTAD (dopant-free), which confirms that the CZTPA-2 device has less interfacial recombination and a higher PCE.

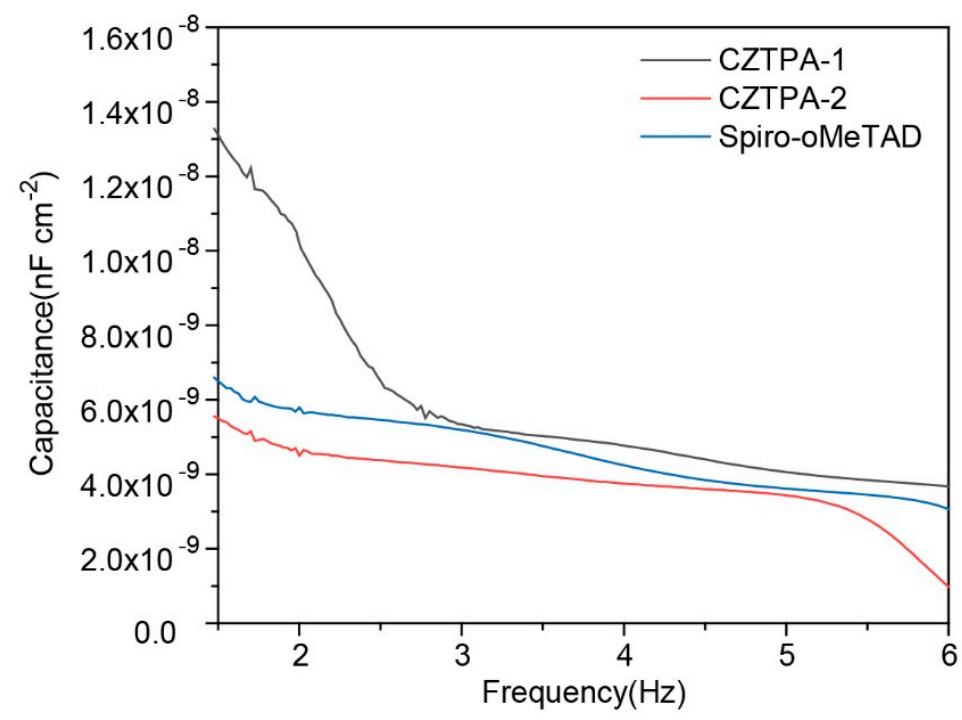

Figure 6. Capacitance versus frequency based on the devices of $\mathrm{ITO} / \mathrm{SnO}_{2} /$ perovskite/HTMs/Au.

\section{Conclusions}

Two new and low-cost hole transporting materials based on a carbazole core were designed and synthesized using a simple synthesis process. CZTPA-2 (dopant-free) achieved the best performance, with a PCE of $11.79 \%$, a $J_{\mathrm{sc}}$ of $21.80 \mathrm{~mA} / \mathrm{cm}^{2}$, a $V_{\mathrm{oc}}$ of $0.99 \mathrm{~V}$, and a FF of $54.59 \%$, which was slightly higher than that of Spiro-OMeTAD (dopant-free) and CZTPA-1 (dopant-free) and attributed to its higher hole transport mobility. The PL spectra, scanning electron microscopy images, and photoelectric properties indicate that CZTPA-2 with a longer alkyl chain has better optoelectrical properties. The CZTPA-2 (dopant-free) device also had the best stability, which remained at $89.7 \%$ of its original PCE after $400 \mathrm{~h}$ compared to CZTPA-1 and Spiro-OMeTAD (dopant-free). Besides this, its hole transport layer thickness is $35 \mathrm{~nm}$. Therefore, we think that CZTPA-2 can also be used to modify an interface when compared to traditional HTMs (above $100 \mathrm{~nm}$ ). The device based on CZTPA-2 exhibited good stability. We conclude that a longer alkyl chain may promote solubility and enhance the perovskite layer's crystallinity.

Supplementary Materials: The following are available online at http://www.mdpi.com/2079-4991/9/7/935/s1, Figure S1: ${ }^{1} \mathrm{H}$ NMR spectrum for compound 1. Figure S2: ${ }^{1} \mathrm{H}$ NMR spectrum for compound 2. Figure S3: ${ }^{1} \mathrm{H}$ NMR spectrum for CZTPA-1. Figure S4: ${ }^{1} \mathrm{H}$ NMR spectrum for CZTPA-2. Figure S5: ${ }^{13} \mathrm{C}$ NMR spectrum for CZTPA-1. Figure S6: ${ }^{13} \mathrm{C}$ NMR spectrum for CZTPA-2. Figure S7: AFM images ( $\left.5 \mu \mathrm{m} \times 5 \mu \mathrm{m}\right)$ of CZTPA-2 (a) and CZTPA-1 (b) films. 
Author Contributions: K.W. conceived the idea; K.W. designed the experiment; H.C. conducted device fabrication; X.G. and T.N. helped in device measurement; K.W. conducted device fabrication and data collection and wrote the manuscript; K.W. and S.W. revised the manuscript; Writing—review \& editing, H.W.; Supervision, H.W.

Funding: This work was supported by the Key Technologies R\&D Plan Projects of Guangdong Province (Nos. 2015B010127013, 2016B010124004, 2017B010112003), by the Science and Technologies Plan Projects of Guangzhou City (Nos.201604046021, 201905010001), and by the Science and Technology Development Special Fund Projects of Zhongshan City (Nos. 2017F2FC0002, 2017A1009).

Conflicts of Interest: The authors declare no conflicts of interest.

\section{References}

1. Kojima, A.; Teshima, K.; Shirai, Y.; Miyasaka, T. Organometal Halide Perovskites as Visible-Light Sensitizers for Photovoltaic Cells. J. Am. Chem. Soc. 2009, 131, 6050-6051. [CrossRef]

2. Michael, M.; Lee, J.L.T.T. Efficient Hybrid Solar Cells Based on Meso-Superstructured Organometal Halide Perovskites. Science 2012, 338, 643-647.

3. Kim, H.; Lee, C.; Im, J.; Lee, K.; Moehl, T.; Marchioro, A.; Moon, S.; Humphry-Baker, R.; Yum, J.; Moser, J.E.; et al. Lead Iodide Perovskite Sensitized All-Solid-State Submicron Thin Film Mesoscopic Solar Cell with Efficiency Exceeding 9\%. Sci. Rep. 2012, 2, 591. [CrossRef] [PubMed]

4. Laura Calil, S.K.M.G. Hole-Transport Materials for Perovskite Solar Cells. Angew. Chem. Int. Ed. 2016, 55, 14522-14545.

5. Bi, D.; Tress, W.; Dar, M.I.; Gao, P.; Luo, J.; Renevier, C.M.; Schenk, K.; Abate, A.; Giordano, F.; Correa Baena, J.; et al. Efficient luminescent solar cells based on tailored mixed-cation perovskites. Sci. Adv. 2016, 2, 1501170-1501176. [CrossRef] [PubMed]

6. NREL Best Research Cell Efficiency Chart. Available online: https://www.nrel.gov/pv/assets/pdfs/bestresearch-cell-efficiencies-190416.pdf (accessed on 27 June 2019).

7. Bach, U.; Lupo, D.; Comte, P.; Moser, J.E.; Weissortel, F.; Salbeck, J.; Spretzer, H.; Gratzel, M. Solid-state dye-sensitized mesoporous $\mathrm{TiO} 2$ solar cells with high photon-to-electron conversion efficiencies. Nature 1998, 395, 583-585. [CrossRef]

8. Jeon, N.J.; Lee, H.G.; Kim, Y.C.; Seo, J.; Noh, J.H.; Lee, J.; Seok, S.I. o-Methoxy Substituents in Spiro-OMeTAD for Efficient Inorganic-Organic Hybrid Perovskite Solar Cells. J. Am. Chem. Soc. 2014, 136, 7837-7840. [CrossRef]

9. Gatti, T.; Menna, E.; Meneghetti, M.; Maggini, M.; Petrozza, A.; Lamberti, F. The Renaissance of fullerenes with perovskite solar cells. Nano Energy 2017, 41, 84-100. [CrossRef]

10. Wang, R.; Mujahid, M.; Duan, Y.; Wang, Z.; Xue, J.; Yang, Y. A Review of Perovskites Solar Cell Stability. Adv. Funct. Mater. 2019, 1808843. [CrossRef]

11. Castro, E.; Murillo, J.; Fernandez-Delgado, O.; Echegoyen, L. Progress in fullerene-based hybrid perovskite solar cells. J. Mater. Chem. C 2018, 6, 2635-2651. [CrossRef]

12. Christians, J.A.; Fung, R.C.M.; Kamat, P.V. An Inorganic Hole Conductor for Organo-Lead Halide Perovskite Solar Cells. Improved Hole Conductivity with Copper Iodide. J. Am. Chem. Soc. 2014, 136, 758-764. [CrossRef] [PubMed]

13. Kim, J.H.; Liang, P.; Williams, S.T.; Cho, N.; Chueh, C.; Glaz, M.S.; Ginger, D.S.; Jen, A.K.Y. High-Performance and Environmentally Stable Planar Heterojunction Perovskite Solar Cells Based on a Solution-Processed Copper-Doped Nickel Oxide Hole-Transporting Layer. Adv. Mater. 2015, 27, 695-701. [CrossRef] [PubMed]

14. Qin, P.; Tanaka, S.; Ito, S.; Tetreault, N.; Manabe, K.; Nishino, H.; Nazeeruddin, M.K.; Gr tzel, M. Inorganic hole conductor-based lead halide perovskite solar cells with $12.4 \%$ conversion efficiency. Nat. Commun. 2014, 5, 3834. [CrossRef] [PubMed]

15. Heo, J.H.; Im, S.H.; Noh, J.H.; Mandal, T.N.; Lim, C.; Chang, J.A.; Lee, Y.H.; Kim, H.; Sarkar, A.; Nazeeruddin, M.K.; et al. Efficient inorganic-organic hybrid heterojunction solar cells containing perovskite compound and polymeric hole conductors. Nat. Photonics 2013, 7, 486-491. [CrossRef]

16. Huang, X.; Zhao, Z.; Cao, L.; Chen, Y.; Zhu, E.; Lin, Z.; Li, M.; Yan, A.; Zettl, A.; Wang, Y.M.; et al. High-performance transition metal-doped Pt3Ni octahedra for oxygen reduction reaction. Science 2015, 348, 1230-1234. [CrossRef] 
17. Edri, E.; Kirmayer, S.; Cahen, D.; Hodes, G. High Open-Circuit Voltage Solar Cells Based on Organic?-CInorganic Lead Bromide Perovskite. J. Phys. Chem. Lett. 2013, 4, 897-902. [CrossRef] [PubMed]

18. Cabau, L.; Garcia-Benito, I.; Molina-Ontoria, A.; Montcada, N.F.; Martin, N.; Vidal-Ferran, A.; Palomares, E. Diarylamino-substituted tetraarylethene (TAE) as an efficient and robust hole transport material for $11 \%$ methyl ammonium lead iodide perovskite solar cells. Chem. Commun. 2015, 51, 13980-13982. [CrossRef]

19. Li, H.; Fu, K.; Hagfeldt, A.; Gr tzel, M.; Mhaisalkar, S.G.; Grimsdale, A.C. A Simple 3,4-Ethylenedioxythiophene Based Hole-Transporting Material for Perovskite Solar Cells. Angew. Chem. Int. Ed. 2014, 53, 4085-4088. [CrossRef]

20. Li, H.; Fu, K.; Boix, P.P.; Wong, L.H.; Hagfeldt, A.; Gr tzel, M.; Mhaisalkar, S.G.; Grimsdale, A.C. Hole-Transporting Small Molecules Based on Thiophene Cores for High Efficiency Perovskite Solar Cells. ChemSusChem 2014, 7, 3420-3425. [CrossRef]

21. Park, S.H.; Roy, A.; Beaupr, S.; Cho, S.; Coates, N.; Moon, J.S.; Moses, D.; Leclerc, M.; Lee, K.; Heeger, A.J. Bulk heterojunction solar cells with internal quantum efficiency approaching 100\%. Nat. Photonics 2009, 3, 297-302. [CrossRef]

22. Qin, R.; Li, W.; Li, C.; Du, C.; Veit, C.; Schleiermacher, H.; Andersson, M.; Bo, Z.; Liu, Z.; Ingana s, O.; et al. A Planar Copolymer for High Efficiency Polymer Solar Cells. J. Am. Chem. Soc. 2009, 131, 14612-14613. [CrossRef] [PubMed]

23. Blouin, N.; Leclerc, M. Poly(2,7-carbazole)s: Structure-Property Relationships. Acc. Chem. Res. 2008, 41, 1110-1119. [CrossRef] [PubMed]

24. Blouin, N.; Michaud, A.; Leclerc, M. A Low-Bandgap Poly(2,7-Carbazole) Derivative for Use in High-Performance Solar Cells. Adv. Mater. 2007, 19, 2295-2300. [CrossRef]

25. Zhu, X.D.; Ma, X.J.; Wang, Y.K.; Li, Y.; Gao, C.H.; Wang, Z.K.; Jiang, Z.Q.; Liao, L.S. Hole-Transporting Materials Incorporating Carbazole into Spiro-Core for Highly Efficient Perovskite Solar Cells. Adv. Funct. Mater. 2018, 29, 1807094. [CrossRef]

26. Chen, Z.; Li, H.; Zheng, X.; Zhang, Q.; Li, Z.; Hao, Y.; Fang, G. Low-Cost Carbazole-Based Hole-Transport Material for Highly Efficient Perovskite Solar Cells. ChemSusChem 2017, 10, 3111-3117. [CrossRef] [PubMed]

27. Xu, B.; Sheibani, E.; Liu, P.; Zhang, J.; Tian, H.; Vlachopoulos, N.; Boschloo, G.; Kloo, L.; Hagfeldt, A.; Sun, L. Carbazole-Based Hole-Transport Materials for Efficient Solid-State Dye-Sensitized Solar Cells and Perovskite Solar Cells. Adv. Mater. 2014, 26, 6629-6634. [CrossRef] [PubMed]

28. Abate, A.; Paek, S.; Giordano, F.; Correa-Baena, J.P.; Saliba, M.; Gao, P.; Matsui, T.; Ko, J.; Zakeeruddin, S.M.; Dahmen, K.H.; et al. Silolothiophene-linked triphenylamines as stable hole transporting materials for high efficiency perovskite solar cells. Energy Environ. Sci. 2015, 8, 2946-2953. [CrossRef]

29. Yin, X.; Guan, L.; Yu, J.; Zhao, D.; Wang, C.; Shrestha, N.; Han, Y.; An, Q.; Zhou, J.; Zhou, B.; et al. One-step facile synthesis of a simple carbazole-cored hole transport material for high-performance perovskite solar cells. Nano Energy 2017, 40, 163-169. [CrossRef]

30. Zimmermann, I.; Urieta-Mora, J.; Gratia, P.; Arag, J.; Grancini, G.; Molina-Ontoria, A.N.; Ort, E.; Mart n, N.; Nazeeruddin, M.K. High-Efficiency Perovskite Solar Cells Using Molecularly Engineered, Thiophene-Rich, Hole-Transporting Materials: Influence of Alkyl Chain Length on Power Conversion Efficiency. Adv. Funct. Mater. 2017, 7, 1601674. [CrossRef]

31. Wang, Y.; Su, T.; Tsai, H.; Wei, T.; Chi, Y. Spiro-Phenylpyrazole/Fluorene as Hole-Transporting Material for Perovskite Solar Cells. Sci. Rep. 2017, 7, 7859-7867. [CrossRef]

(C) 2019 by the authors. Licensee MDPI, Basel, Switzerland. This article is an open access article distributed under the terms and conditions of the Creative Commons Attribution (CC BY) license (http://creativecommons.org/licenses/by/4.0/). 